\title{
Study on a Wind Velocity Measurement Method at High Positions with Drones
}

\author{
Nobuhiro Kishigaki ${ }^{\mathrm{a},}{ }^{,}$, Hiromitsu Ijichi ${ }^{\mathrm{a}}$, Keiichi Yoshino ${ }^{\mathrm{a}}$, Teruhisa Tatsuoka ${ }^{\mathrm{a}}$ \\ ${ }^{a}$ R\&D Dept. TEPCO Research Institute, Tokyo Electric Power Company Holdings, Inc. \\ 4-1, Egasaki-cho, Tsurumi-ku, Yokohama, 230-8510, Japan \\ *Corresponding Author: kishigaki.nobuhiro@tepco.co.jp
}

\begin{abstract}
Work at high positions, such as the tops of transmission towers, in strong winds with more than $10 \mathrm{~m} / \mathrm{s}$ of 10 minutes average wind velocity is prohibited by the Ordinance on Industrial Safety and Health in Japan. Therefore, to judge whether or not work can be performed, we studied measuring wind velocity at high positions using a drone and inclinometer, which is a simple method that does not require extra cost or labor. This method enables safe and easy measurement of approximate wind velocity at high positions.
\end{abstract}

Keywords: drone, wind velocity, tilt angle.

\section{Introduction}

At present, electric power facilities exposed in atmospheric environments are mainly inspected visually by humans, and when checking transmission towers, operators have to work at the high positions from dozens of meters to hundreds of meters high above the ground. However, in Japan, work at heights of $2 \mathrm{~m}$ or more and in strong winds with more than $10 \mathrm{~m} / \mathrm{s}$ of 10 minutes average wind velocity is prohibited by the Ordinance on Industrial Safety and Health $^{(1)}$.

Therefore, the wind velocity at high positions is estimated before work from the wind velocity measured on the ground and the movement of branches and leaves on trees nearby transmission towers, however there is a considerable error.

Although there are methods for accurately measuring wind velocity at high positions, such as installing an anemometer on a steel tower or installing Doppler Sodar on the ground, they cost a lot and are not realistic. A low-cost and convenient method for measuring wind velocity at high positions is needed.

A method that uses drones (unmanned aerial vehicles: UAVs), which have become more and more sophisticated and less expensive in recent years, is also being studied ${ }^{(2,3)}$. An airflow sensor is installed on the drone to measure wind velocity. However, it has been found that the airflow from the drone's propeller may affect the results of wind velocity measurement ${ }^{(3)}$. Another method of calculating the wind direction and velocity from the time-series attitude data of the drone in flight has also been proposed ${ }^{(4-6)}$. However, this method requires a complicated process: the attitude data is collected from the flight controller, and wind direction and velocity are then calculated based on the collected attitude data.

Therefore, we studied a more convenient and inexpensive method of measuring wind velocity at high positions using a drone and inclinometer.

\section{Methods}

\subsection{Method of measuring wind velocity at High Positions}

In recent years, drones have become more sophisticated and less expensive, and they can check their position based on GPS signals, and they can stand still while hovering stably in a fixed position.

Fig. 1 shows the drone's tilt against the wind. In windy conditions, they hover while tilting upwind to resist wind pressure and maintain their position. Fig. 2 shows schematic diagram of inclinometer and camera installed in drone. While hovering, the drone body tilts according to the wind velocity, the drone's camera reads the tilt angle of the drone from the inclinometer installed in the front of the 
camera, and the wind velocity based on the tilt angle can be estimated.

\subsection{Installing the inclinometer on the drone}

Fig. 3 shows the drone used in the experiment. The drone is expected to measure wind velocities up to $10 \mathrm{~m} / \mathrm{s}$, the reference value for judging whether or not work at high positions can be performed. In addition, a person may be assumed to carry the drone into a mountainous area on foot. Therefore, it is desirable for drones to be as small and lightweight as possible. However, drones with larger bodies tend to have higher wind resistant performance. Therefore, taking both these factors into consideration, the DJI PHANTOM 4 ADVANCED, which weighs $1,368 \mathrm{~g}$ and has a diagonal length of $350 \mathrm{~mm}$ (excluding propellers) was chosen for this study.

Fig. 4 shows the inclinometer used in the experiment. Considering that the angle of tilt may fluctuate due to vibration during wind velocity measurement, the LEV-O-GAGE $45^{\circ}$ was selected for the inclinometer. This inclinometer has a graded scale tube with brass ball inside and filled with a damping liquid to suppress the movement of the brass ball to some extent.

Fig. 5 shows the position of the installed inclinometer. The inclinometer was installed in front of the camera so that it would not interfere with other sensors to control the drone.

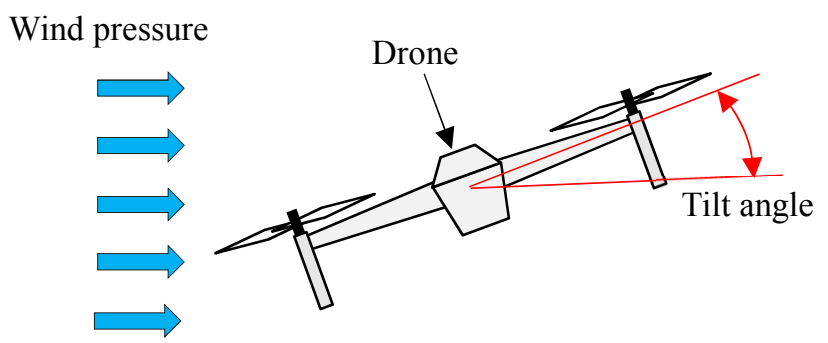

Fig. 1. Drone attitude and tilt angle against wind pressure.

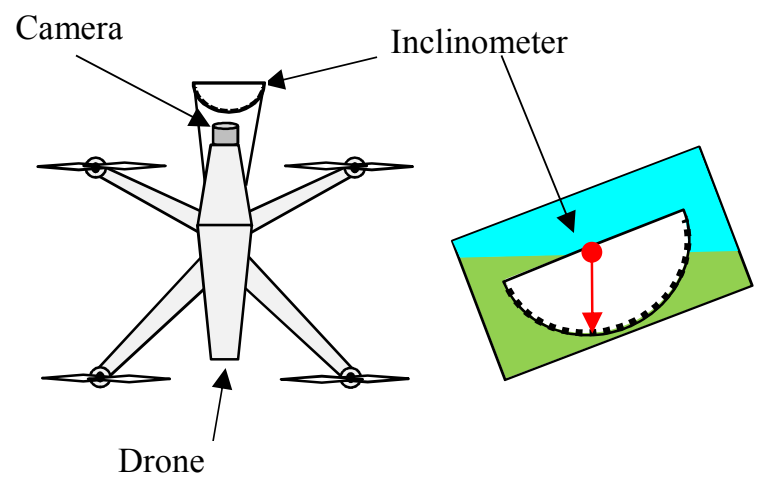

Fig. 2. Schematic diagram of inclinometer and camera installed in drone.

\subsection{Blower for experiment}

The tilt angle of a drone relative to the wind velocity differs depending on the model. In order to estimate wind velocity from the tilt angle of a drone at high positions, it is necessary to carry out experimentation beforehand to ascertain the tilt angle of the model relative to the wind velocity. For the experiment to evaluate the relationship between the tilt angles with each drone, a commercial blower was installed indoors away from the influence of natural wind. Previous research ${ }^{(7)}$ in which a blower was used was also used as a reference when carrying out the experiment.

The blower used was the Dynamic Fan DF-500 CA manufactured by Nichido Ind. Co., Ltd. (blade diameter $500 \mathrm{~mm}, 8$ blades, rated voltage $100 \mathrm{~V}, 580 \mathrm{~W}$, maximum wind speed $12.2 \mathrm{~m} / \mathrm{s}$, maximum air volume $150 \mathrm{~m}^{3} / \mathrm{min}$ ). This blower was installed at the end of a warehouse with a width of $12.8 \mathrm{~m}$, length of $20.6 \mathrm{~m}$, and height of $8.2 \mathrm{~m}$. The outlet was directed toward the center of the laboratory, and the rotating shaft of the blower was made parallel with the floor. In order to understand the spatial distribution of the wind velocity, we made sure that the height of the rotating shaft was $800 \mathrm{~mm}$, and measured the velocity of wind from the blower using the PC-51 D anemometer manufactured by Sato Keiryoki Mfg. Co., Ltd. Since the wind velocity fluctuated a little, the average wind velocity for five seconds was measured. The measuring points were on the line extending from the rotating shaft of the blower. Fig. 6 shows the measurement results of wind velocity distribution on the line extending from the rotating shaft of the blower.

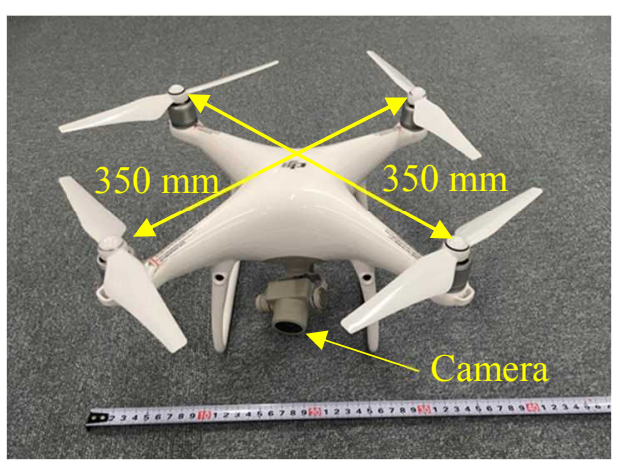

Fig. 3. Drone and installed camera.

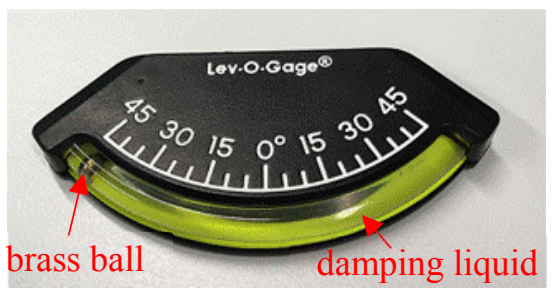

Fig. 4. Inclinometer installed on drone. 


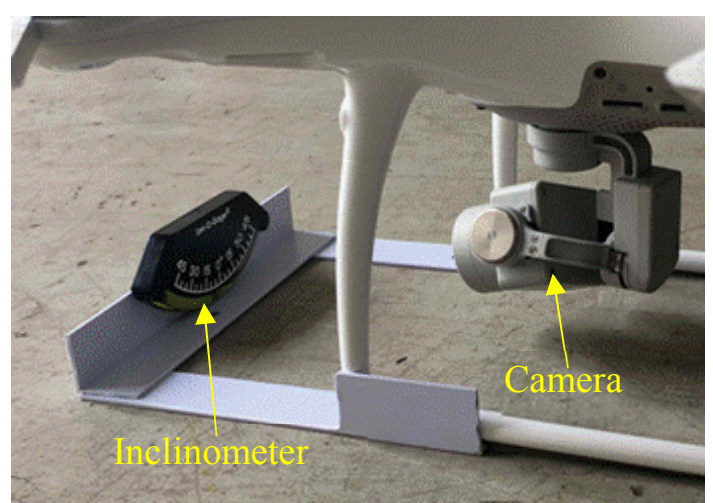

Fig. 5. Position of the installed inclinometer and camera.

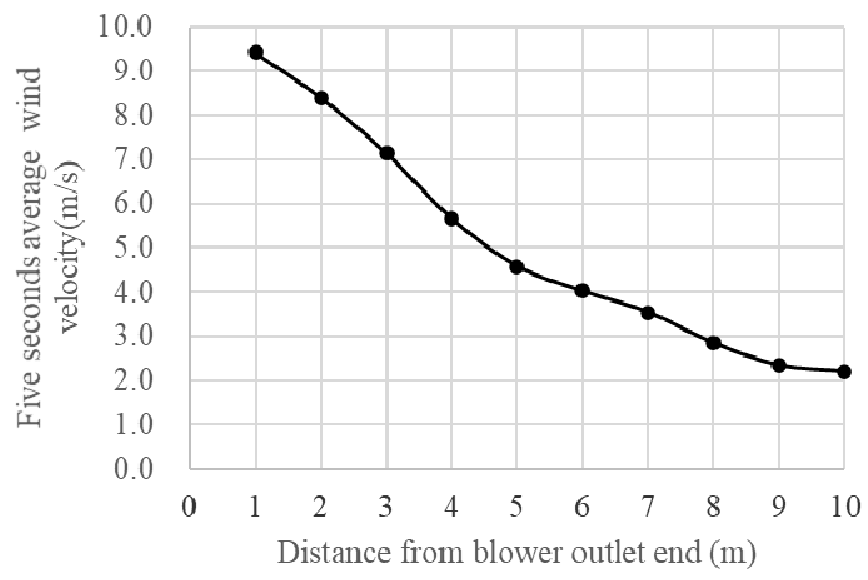

Fig. 6. Wind velocity distribution on the line extending from the rotating shaft of the blower.

The wind velocity decreased as the distance from the blower increased. Fig. 7 and Fig. 8 show the spatial distribution of the wind velocity on vertical planes normal to the line extending from the rotating shaft of the blower which are $2 \mathrm{~m}$ and $5 \mathrm{~m}$ from the outlet end of the blower. At a position closer to the blower, the wind velocity on the line extending from the rotating shaft of the blower is large, and the gradient of the wind velocity distribution around it is also large. On the other hand, the farther the position is from the blower, the more the spatial distribution of the wind velocity become uniform. In Fig. 7, the wind velocity is the highest ( 8 to $9 \mathrm{~m} / \mathrm{s}$ ) in the range where the vertical distance from the line extending from the rotating shaft of the blower is less than $0.2 \mathrm{~m}$. Therefore, when measuring the tilt angle of the drone in the area with high wind velocity, the drone should keep its center as close as possible to the line extending from the rotating shaft of the blower while hovering.

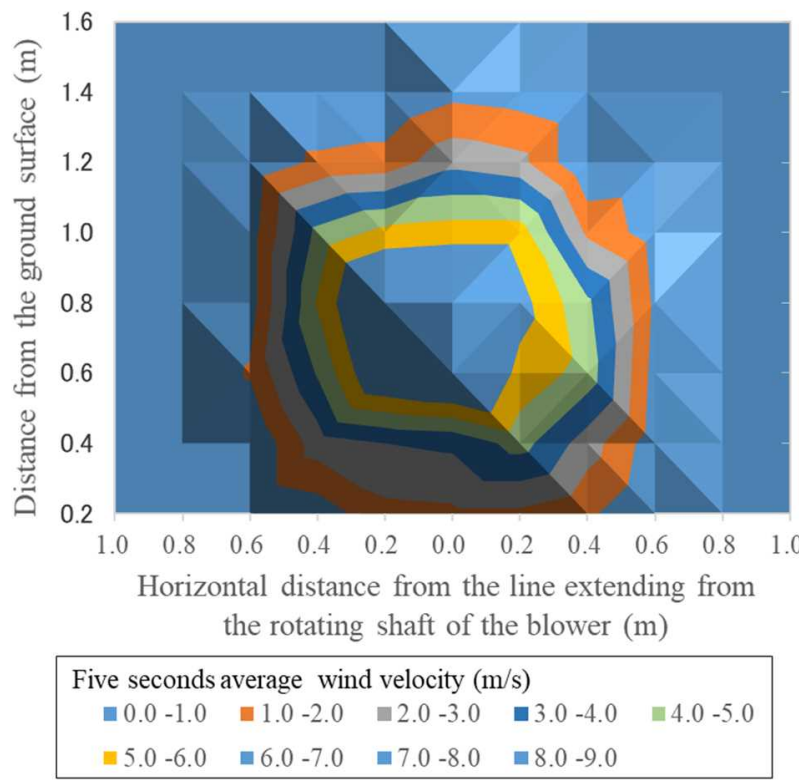

Fig. 7. Wind velocity distribution at $2 \mathrm{~m}$ from the blower outlet

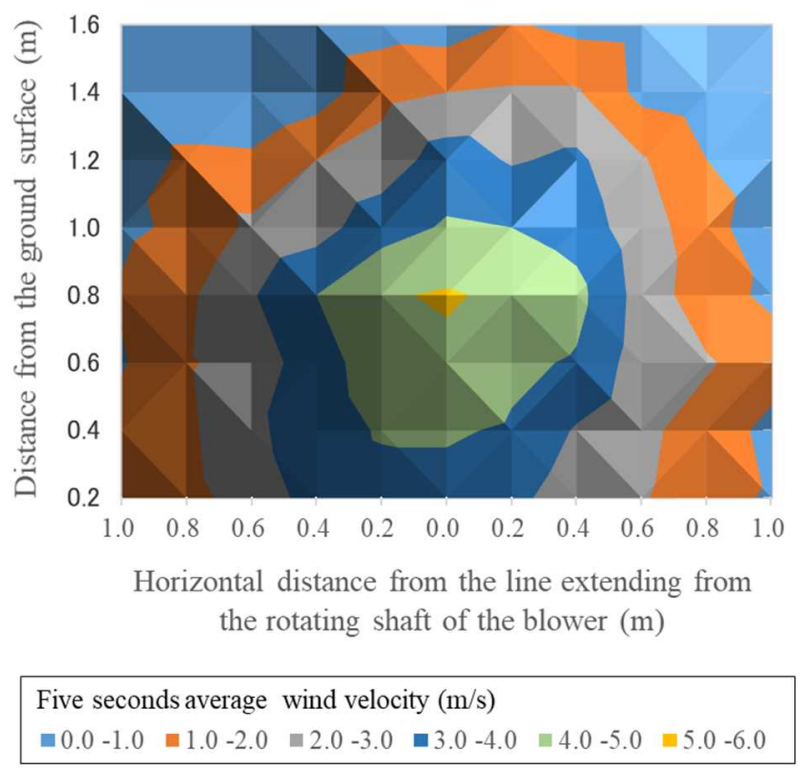

Fig. 8. Wind velocity distribution at $5 \mathrm{~m}$ from the blower outlet.

\subsection{Measurement of the tilt angle of drone}

The DJI PHANTOM4 ADVANCED was hovered on the extension of the blower rotation axis at the height of 0.8 $\mathrm{m}$ from the ground. The line extending from the rotating shaft of the blower was orthogonalized with the rolling axis of the drone. In other words, the side of the drone faced the direction of the blower. While the drone was hovering, the tilt angle of the drone in the rolling direction captured by the camera was read visually. Fig. 9 shows an image of tilt angle view of inclinometer captured by camera installed on drone. 


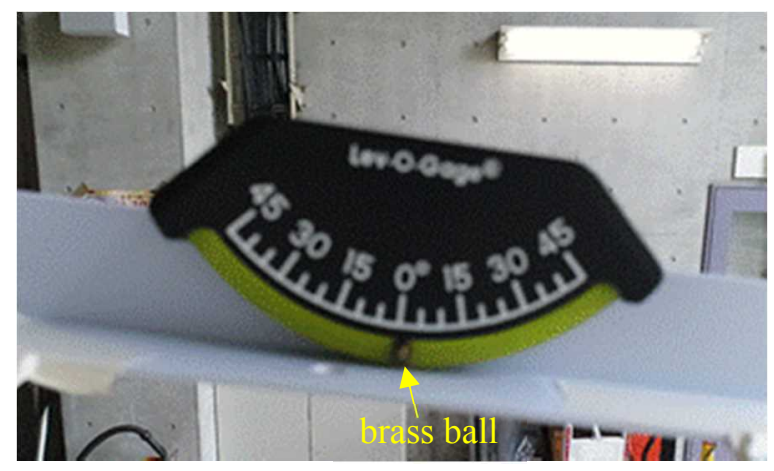

Fig. 9. An image of tilt angle view of inclinometer captured by camera installed on drone.

\section{Results and Discussion}

Fig. 10 shows the tilt angle of the drone relative to the wind velocity measured. According to Fig. 10, the tilt angle of the drone increases as the wind velocity increases. It is considered that the approximate wind velocity in the high positions can be easily measured using the correlation between wind velocity and tilt angle. This measurement method does not require any special equipment. The wind speed can be measured simply by installing an inclinometer in front of the drone's camera.

Because the wind from the blower fluctuated slightly, the brass ball in the inclinometer also vibrated to some extent. Since the median value of the oscillating angle range was read, the result may include some errors.

An analog scale inclinometer with increments in 5-degree intervals was used in this experiment. This is the reason why the inclinometer weighs only $20 \mathrm{~g}$, which is extremely light. However, it is possibly better to use a digital inclinometer so that the tilt angle can be read more easily. Even though, there is a high possibility that the displayed value will not stable. Furthermore, since the weight of such an inclinometer is expected to be heavier, it is also necessary to consider the size and payload of the drone to be used.

The DJI PHANTOM 4 ADVANCED has a wind resistance of $10 \mathrm{~m} / \mathrm{s}$, in the vicinity of the outlet of the blower, it could not stay stationary at a specific distance because it was carried downwind. It is desirable to use a drone with a high wind resistance exceeding $10 \mathrm{~m} / \mathrm{s}$.

The drone weighs $1,368 \mathrm{~g}$, which is somewhat heavy for a person to carry into a mountainous area on foot. Lighter drones are more preferable and convenient to use. However, the drone must be installed with an inclinometer, so the drone requires a certain payload.

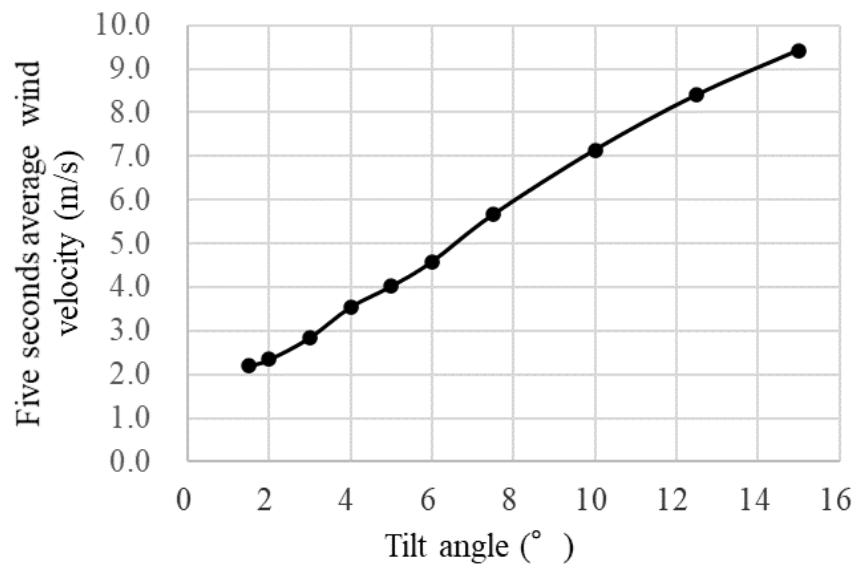

Fig. 10. Correlation between wind velocity and tilt angle of drone.

When the drone model is changed, the tilt angle of the drone relative to the wind velocity also changes, so it is necessary to obtain a calibration curve for the relationship between tilt angle and wind velocity for each model.

\section{Conclusions}

The tilt angle of a drone relative to wind velocity was measured with a drone equipped with an inclinometer. We consider that wind velocity at high positions can be measured in a simplified manner using the correlation between wind velocity and tilt angle of drone. This measurement method does not require any special equipment, so it is very convenient when wind velocity measurement is required in the field work.

\section{References}

(1) The Ordinance on Industrial Safety and Health

(2) Sam Prudden, A Fisher, Matthew Marino, Abdulghani Mohamed, Simon Watkins, Graham Wild: "Measuring wind with Small Unmanned Aircraft Systems", Journal of Wind Engineering and Industrial Aerodynamics, Vol.176, pp.197-210, 2018

(3) Kenta Takaya, Valeri Kroumov, Hiroshi Ohta, Keishi Shibayama: "Measuring Wind Speed Using the Internal Stabilization System of a Quadrotor Drone", 2021 IEEJ annual meeting, No. 3-063, pp.94-95, 2021

(4) Neumann Patrick, Bartholmai Matthias: "Real-time wind estimation on a micro unmanned aerial vehicle using its inertial measurement unit", Sensors and Actuators. A. Physical, Vol.235 pp.300-310, 2015

(5) Kansuke Sasaki, Minoru Inoue, Hiromi Kojima, Kawami Hirofumi, Machida Shunichi, Yutaka 
Watanabe, Etsurou Natori, Nobuyuki Fukuda, Naoyuki Hirasaka: "Development upper Air Observation Method Using UAV (Unmanned Aerial Vehicle)", Disaster Prevention Research Institute Annuals, No. 59B, pp.395-402, 2016 (in Japanese)

(6) Simma Magdalena, Mjoen Havard, Bostrom Tobias: "Measuring Wind Speed Using the Internal Stabilization System of a Quadrotor Drone", Drones, Vol.4, No. 2, pp.23, 2020

(7) Kazuhiko Yamazaki, Akiko Maeda, Chiho Okubo: "Properties of Blower for the Experiments of Wind Effects", Prospectus of Faculty of Human Life Sciences, Jissen Women's University, No. 50, pp. 187-190, 2013 